\title{
PARAMETRIC METHODS FOR POLE ASSIGNMENT
}

\author{
A. Varga \\ DLR Oberpfaffenhofen \\ German Aerospace Research Establishment \\ Institute of Robotics and System Dynamics \\ D-82234 Wessling, Germany \\ fax: +49-8153-281441, e-mail: Andreas.Varga@dlr.de
}

Keywords : Numerical methods, feedback stabilization, linear systems.

\section{Abstract}

The non-redundant parametrization of the pole assignment problem for a $n$-th order system with $m$ inputs allows to express the solution of the problem in term of $n(m-1)$ free parameters. These parameters can be used to fulfill additional requirements on the closed-loop system as for instance minimum norm feedback gain matrix, well conditioned eigenvector set, maximum stability radius. One of reliable numerical methods for pole assignment is the so-called Schur method. An extension of this method is proposed which computes the solution of the pole assignment problem corresponding to a non-redundant parameter set. Several possibilities are further investigated to compute minimum norm feedback matrices. An improved approach to compute minimum Frobenius-norm feedback relying on a redundant parametrization is also discussed.

\section{Introduction}

We consider the following eigenvalue assignment problem (EAP): given the controllable matrix pair $(A, B)$, where $A \in \mathbb{R}^{n, n}$ and $B \in \mathbb{R}^{n, m}$, determine the feedback matrix $F \in \mathbb{R}^{m, n}$ such that the closed-loop state matrix $A+B F$ has all its eigenvalues at desired locations $\Gamma=\left\{\lambda_{1}, \ldots, \lambda_{n}\right\}$ in the complex plane. We assume that $\Gamma$ is symmetric with respect to the real axis. This assumption guarantees that the resulting $F$ is real. There exist several numerically stable algorithms which can be used to solve the $\operatorname{EAP}[7,9,6]$. All these methods are based on the orthogonal controllability staircase form of the pair $(A, B)[11]$. An alternative to these methods is the so-called Schur method proposed by Varga [12] which uses the real Schur form (RSF) of the matrix to accomplish the eigenvalue assignment. Although computationally more involved than the previous ones, the Schur method has the attractive feature to allow a partial pole assignment, i.e. it is possible to alter only those eigenvalues of $A$ which are unsatisfactory for the closed-loop system dynamics and to keep unmodified the rest of eigenvalues. The Schur approach has been extended to generalized state-space systems [16] as well as to periodic systems [10]. Moreover the Schur method has been adapted to compute various coprime factorizations of rational matrices $[14,15]$.

The solution of the EAP can be generally expressed in terms of $n(m-1)$ free parameters [8], thus in the multiinput case $(m>1)$ this freedom can be exploited to fulfill additional requirements. In [3] the parametric freedom is explicitly addressed by trying to minimize the sensitivity of closed-loop eigenvalues. The minimization of the norm of the feedback matrix assigning a set of eigenvalues has been considered in [4], where an explicit gradient search is performed. An alternative approach has been proposed in [8] by extending the algorithm of [9] such that the computed solution is dependent of a prescribed parameter set. In all these methods an over-parametrization of the problem is usually present.

In this paper we discuss an extension of the Schur method of [12] to cope with explicit parametric information. The proposed extension uses exactly $n(m-1)$ free parameters to perform the assignment of a complete set of $n$ eigenvalues. The method can also be employed to assign a set of $k<n$ eigenvalues by modifying only $k$ eigenvalues of $A$ and keeping unaltered the rest of $n-k$ eigenvalues and by using exactly $k(m-1)$ free parameters for this purpose. This partial pole assignment is useful for example in solving efficiently minimum norm feedback stabilization problems. The proposed parametric Schur method relies on explicit non-redundant parametrizations of EAPs of systems of orders at most 2.

Several possibilities are also investigated to compute minimum norm state-feedback matrices for eigenvalue assignment or feedback stabilization of systems of order at most 2. For arbitrary order systems, an improved approach to compute minimum Frobenius-norm feedback relying on a redundant parametrization is proposed. 


\section{Parametric Schur Method}

The Schur method relies on a very simple fact explained below. Consider the matrix $A$ assumed to be already in RSF and partitioned conformally with $B$ as follows

$$
A=\left[\begin{array}{cc}
A_{11} & A_{12} \\
0 & A_{22}
\end{array}\right], \quad B=\left[\begin{array}{c}
B_{1} \\
B_{2}
\end{array}\right],
$$

where $A_{22}$ is the last diagonal block of order $n_{1}=1$ or 2. Because the pair $(A, B)$ is controllable, it follows that $\left(A_{22}, B_{2}\right)$ is controllable too. Thus, given a symmetric set $\Gamma_{1} \subset \Gamma$ of $n_{1}$ complex values, we can determine $K_{1}$ such that $\Lambda\left(A_{22}+B_{2} K_{1}\right)=\Gamma_{1}$. With the partial feedback $F_{1}=\left[\begin{array}{ll}0 & K_{1}\end{array}\right]$ we obtain

$$
A+B F_{1}=\left[\begin{array}{cc}
A_{11} & A_{12}+B_{1} K_{1} \\
0 & A_{22}+B_{2} K_{1}
\end{array}\right]
$$

and thus the matrix $A+B F_{1}$ has $n-n_{1}$ unmodified eigenvalues $\Lambda\left(A_{11}\right)$ and $n_{1}$ new eigenvalues at desired positions in $\Gamma_{1}$. Moreover, $A+B F_{1}$ is further in RSF. By reordering the diagonal blocks of $A+B F_{1}$ with the help of an orthogonal similarity transformation to get $A_{1}=Q_{1}^{T}(A+B F) Q_{1}$, it is possible to bring in the last position of $A_{1}$ another block containing $n_{2}$ unmodified eigenvalues of the original $A$. By using a new partial feedback $F_{2}=\left[\begin{array}{ll}0 & K_{2}\end{array}\right] Q_{1}^{T}$ the matrix $A+B \widetilde{F}$ with $\widetilde{F}=F_{1}+F_{2}$, has $n-n_{1}-n_{2}$ unmodified eigenvalues of the original $A$ and $n_{1}+n_{2}$ eigenvalues at desired new positions. This process can be repeated until all eigenvalues of $A$ are modified. Thus the final feedback $F$ is obtained as a sum of partial feedbacks

$$
F=\sum F_{i}
$$

where each partial feedback $F_{i}$ assigns one or two eigenvalues. The number of partial feedbacks in the above sum depends on the number of modified blocks (note that occasionally two $1 x 1$ blocks are joined to assign a pair of complex conjugate eigenvalues).

The Schur method can easily cope with the parametric freedom for multivariable pole assignment. For instance, to compute $F_{1}$ it is possible to use $n_{1}(m-1)$ free parameters $p_{i}, i=1, \ldots, n_{1}(m-1)$ in the expression of $K_{1}$ (see next section). Further, $n_{2}(m-1)$ parameters can be used to determine the next partial feedback $F_{2}$ and so on. In this way a total number of $n(m-1)$ parameters can be finally used to determine $F$ which solves the original EAP. If the Schur method is used for a partial pole assignment to modify say only $k$ eigenvalues of $A$ (which are not satisfactory), then this can be done by using $k(m-1)$ parameters for this purpose.

The following pole assignment algorithm is a parameterized version of the procedure of [12]. It allows to perform a partial pole assignment by modifying only those eigenvalues of $A$ which belong to a "bad" region $\mathbb{C}_{b}$ of the complex plane $\mathbb{C}$, keeping the rest of eigenvalues unmodified. In the following procedure we assume that we have given a set of $n(m-1)$ parameters which belongs to a parameter vector $p \in \mathbb{R}^{n(m-1)}$. These parameters can be provided for instance, by an optimizer which tries to ensure certain desirable properties of the resulting feedback $F$ or of the resulting closed-loop pair $(A+B F, B)$. Notice that in case of partial pole assignment not all parameters in $p$ will be used by the algorithm.

\section{Algorithm PSM. Parametric Schur Method.}

1. By using an orthogonal similarity transformation, reduce the matrix $A$ to the RSF

$$
Q^{T} A Q=\left[\begin{array}{cc}
A_{11} & A_{12} \\
0 & A_{22}
\end{array}\right]
$$

where $A_{11} \in \mathbb{R}^{q, q}, Q$ is an orthogonal matrix, $\Lambda\left(A_{11}\right) \subset$ $\mathbb{C} \backslash \mathbb{C}_{b}$ and $\Lambda\left(A_{22}\right) \subset \mathbb{C}_{b}$. Compute $A \leftarrow Q^{T} A Q$ and $B \leftarrow Q^{T} B$; set $F=0$ and $i=0$.

2. If $q=n$, Stop.

3. Let $\alpha$ be the $k \times k$ last diagonal blocks of $A(k=1$ or 2$)$ in RSF and let $\beta$ be the $k \times m$ matrix formed from the last $k$ rows of $\mathrm{B}$. Choose $\Gamma^{\prime} \subset \Gamma$ an appropriate set of $k$ eigenvalues to be assigned. By using the parameters $\left\{p_{i+1}, \ldots, p_{i+k(m-1)}\right\}$ (see next section), determine $\varphi$ such that $\Lambda(\alpha+\beta \varphi)=\Gamma^{\prime}$.

4. Compute $A \leftarrow A+B[0 \varphi], F \leftarrow F+[0 \varphi] Q^{T}$.

5. Compute the orthogonal matrix $\widetilde{Q}$ to reorder the diagonals blocks of $A$ in RSF, such that the last block of $A$ is moved by successive interchanging of diagonal blocks to row position $q+1$. Compute $A \leftarrow \tilde{Q}^{T} A \tilde{Q}, B \leftarrow \tilde{Q}^{T} B$ and $Q \leftarrow Q \tilde{Q}$.

6. Put $q \leftarrow q+k, i \leftarrow i+k(m-1), \Gamma \leftarrow \Gamma \backslash \Gamma^{\prime}$ and go to step 2 .

The usefulness of this algorithm can be judged in conjunction with the use of parameter tuning techniques to determine the partial feedbacks $\varphi$ at Step 3 to achieve "local" feedback performances or the final feedback $F$ to fulfill certain desired "global" characteristics. The case of minimum norm partial feedbacks is discussed in section 4. A typical approach to obtain global feedback performances is to use the above algorithm in conjunction with combinatorial strategies to choose suitable orderings of eigenvalues to be assigned together with global parameter search techniques (see for instance [2]). Several criteria to be optimized by choosing the free parameters are mentioned in [9], as for instance the minimization of the norm of feedback $F$, maximization of the distance to the instability of the closed loop matrix $A+B F$, or the minimization of condition numbers of assigned eigenvalues. To compute parameterized feedback matrices with the PSM Algorithm, explicit feedback parametrizations are necessary for systems of orders at most 2. Such parametrizations are derived in the next section. 


\section{Parametric Pole Assignment: the $1 \times 1$ and $2 \times 2$ Cases}

In this section we consider the parametric EAP for systems of order $n=1$ or $n=2$. The developed formulas can be used in PSM Algorithm to perform parametric pole assignment at Step 3.

The case $n=1$. Let $V$ be an orthogonal matrix to compress the columns of $B$ to a single element, that is,

$$
B V=\left[\begin{array}{llll}
\beta & 0 & \ldots & 0
\end{array}\right]
$$

If $\Gamma=\{\gamma\}$, then for given $p \in \mathbb{R}^{m-1}$ the feedback $F$ which assigns $\gamma$ can be computed as

$$
F(p)^{T}=\left[\begin{array}{llll}
\frac{\gamma-A}{\beta} & p_{1} & \ldots & p_{m-1}
\end{array}\right] V^{T}
$$

Notice that the feedback $F(p)$ in (2) can be expressed as

$$
F(p)=F_{0}+\widetilde{F}(p),
$$

where

$$
F_{0}=V e_{1} \frac{\gamma-A}{\beta}=B^{\#}(\gamma-A)
$$

is the minimum norm feedback to assign the eigenvalue $\gamma$ ( $e_{1}$ is the first column of the identity matrix and $B^{\#}$ is the Moore-Penrose pseudoinverse of $B$ ) and

$$
\widetilde{F}(p)^{T}=\left[\begin{array}{llll}
0 & p_{1} & \ldots & p_{m-1}
\end{array}\right] V^{T}
$$

is a parametric feedback which does not perturb the spectrum of $A$.

The case $n=2$. Consider the singular value decomposition of $B$ as

$$
U^{T} B V=[\Sigma 0],
$$

where $U$ and $V$ are orthogonal matrices and

$$
\Sigma=\left[\begin{array}{cc}
\beta_{1} & 0 \\
0 & \beta_{2}
\end{array}\right]
$$

with $\beta_{1} \geq \beta_{2} \geq 0$. The transformed state matrix becomes

$$
\widetilde{A}=U^{T} A U=\left[\begin{array}{ll}
\alpha_{11} & \alpha_{12} \\
\alpha_{21} & \alpha_{22}
\end{array}\right] .
$$

Because the pair $(A, B)$ is controllable, $\beta_{1}>0$ and either $\beta_{2}>0$ or $\alpha_{21} \neq 0$ if $\beta_{2}=0$. Let $\Gamma=\left\{\gamma_{1}, \gamma_{2}\right\}$ be the set of desired eigenvalues. First we solve the EAP for the reduced pair $(\widetilde{A}, \Sigma)$ by determining the feedback $\widetilde{F}$ such that $\Lambda(\widetilde{A}+\Sigma \widetilde{F})=\Gamma$. Explicit expressions for the entries of the feedback matrix

$$
\widetilde{F}=\left[\begin{array}{ll}
\varphi_{11} & \varphi_{12} \\
\varphi_{21} & \varphi_{22}
\end{array}\right]
$$

can be obtained by solving the equations

$$
\begin{aligned}
\gamma_{1}+\gamma_{2}= & \alpha_{11}+\beta_{1} \varphi_{11}+\alpha_{22}+\beta_{2} \varphi_{22} \\
\gamma_{1} \gamma_{2}= & \left(\alpha_{11}+\beta_{1} \varphi_{11}\right)\left(\alpha_{22}+\beta_{2} \varphi_{22}\right) \\
& -\left(\alpha_{12}+\beta_{1} \varphi_{12}\right)\left(\alpha_{21}+\beta_{2} \varphi_{21}\right)
\end{aligned}
$$

We solve these equations for $\varphi_{11}$ and $\varphi_{12}$ in terms of $\varphi_{21}$ and $\varphi_{22}$. Denoting $\sigma=\gamma_{1}+\gamma_{2}$ and $\pi=\gamma_{1} \gamma_{2}$ we obtain

$$
\begin{aligned}
& \varphi_{11}=\frac{\sigma-\alpha_{11}-\alpha_{22}-\beta_{2} \varphi_{22}}{\beta_{1}} \\
& \varphi_{12}=-\frac{\alpha_{12}}{\beta_{1}}-\frac{\pi-\left(\sigma-\alpha_{22}-\beta_{2} \varphi_{22}\right)\left(\alpha_{22}+\beta_{2} \varphi_{22}\right)}{\beta_{1}\left(\alpha_{21}+\beta_{2} \varphi_{21}\right)}
\end{aligned}
$$

Given the vector of parameters $p \in \mathbb{R}^{2(m-1)}$, we can set $\varphi_{21}=p_{1}$ and $\varphi_{22}=p_{2}$ and thus we can compute

$F$ which assigns $\Gamma$ as

$$
F(p)=V\left[\begin{array}{cc}
\varphi_{1} & \varphi_{2} \\
p_{1} & p_{2} \\
p_{3} & p_{4} \\
\vdots & \vdots \\
p_{2 m-3} & p_{2 m-2}
\end{array}\right] U^{T},
$$

where

$$
\begin{aligned}
\varphi_{1} & =\frac{\sigma-\alpha_{11}-\alpha_{22}-\beta_{2} p_{2}}{\beta_{1}} \\
\varphi_{2} & =-\frac{\alpha_{12}}{\beta_{1}}-\frac{\pi-\left(\sigma-\alpha_{22}-\beta_{2} p_{2}\right)\left(\alpha_{22}+\beta_{2} p_{2}\right)}{\beta_{1}\left(\alpha_{21}+\beta_{2} p_{1}\right)}
\end{aligned}
$$

There exist many other parametrizations of the feedback $F(p)$ in terms of $2(m-1)$ free parameters. The above parametrization has the main advantage that it is valid for all values of $\beta_{2}$. Thus the case $\beta_{2}=0$ is also covered and the expressions are therefore valid in the single-input case too. Moreover if $\Gamma=\Lambda(A)$ the resulting $F(p)=0$ if $p=0$, provided $\alpha_{21} \neq 0$. Notice however that because of the condition $\alpha_{21}+\beta_{2} p_{1} \neq 0$, the above parametrization is not continuous with respect to $p_{1}$. This aspect could lead occasionally to failures of search techniques involving continuous variation of $p_{1}$.

A continuous parametrization can be defined if $\beta_{2} \neq 0$. Let $F_{0}$ be a feedback matrix such that $\Lambda\left(A+B F_{0}\right)=\Gamma$. In particular $F_{0}$ can be taken a minimum norm feedback (see next section). Then we define $F(p)$ in the form (3), where $\widetilde{F}(p)$ can be chosen as

$$
\widetilde{F}(p)=V\left[\begin{array}{c|c}
\Sigma^{-1}(J-\widehat{A}) \\
\hline p_{3} & p_{4} \\
\vdots & \vdots \\
p_{2 m-3} & p_{2 m-2}
\end{array}\right] U^{T},
$$

with $\widehat{A}=U^{T}\left(A+B F_{0}\right) U, \Lambda(J)=\Gamma$ and $J=J\left(p_{1}, p_{2}\right)$ has as entries continuous functions of two parameters $p_{1}$ and $p_{2}$. For instance, if we denote

$$
\widehat{A}=\left[\begin{array}{ll}
\widehat{\alpha}_{11} & \widehat{\alpha}_{12} \\
\widehat{\alpha}_{21} & \widehat{\alpha}_{22}
\end{array}\right]
$$

then we can choose $J$ as $J=Q\left(p_{2}\right)^{-1} \widehat{J}\left(p_{1}\right) Q\left(p_{2}\right)$, where

$$
\widehat{J}\left(p_{1}\right)=\left[\begin{array}{cc}
\widehat{\alpha}_{11}-p_{1} & \widehat{\alpha}_{12}-\frac{p_{1}\left(p_{1}+\widehat{\alpha}_{22}-\widehat{\alpha}_{11}\right)}{\widehat{\alpha}_{21}} \\
\widehat{\alpha}_{21} & \widehat{\alpha}_{22}+p_{1}
\end{array}\right]
$$


and $Q\left(p_{2}\right)$ can be chosen the non-orthogonal matrix

$$
Q\left(p_{2}\right)=\left[\begin{array}{cc}
1 & 0 \\
p_{2} & 1
\end{array}\right]
$$

or even an orthogonal matrix

$$
Q\left(p_{2}\right)=\left[\begin{array}{cc}
\frac{1}{\sqrt{1+p_{2}^{2}}} & \frac{p_{2}}{\sqrt{1+p_{2}^{2}}} \\
-\frac{p_{2}}{\sqrt{1+p_{2}^{2}}} & \frac{1}{\sqrt{1+p_{2}^{2}}}
\end{array}\right]
$$

The above choice ensures that for $p_{1}=0, p_{2}=0 \mathrm{~J}=\widehat{A}$ and thus $\widetilde{F}(0)=0$.

\section{Pole Assignment with Minimum Norm Partial Feedbacks}

The usage of small norm feedback gains are desirable from engineering point of view because usually involve less control efforts. Small gains are also desirable from numerical point of view because of enforcing better numerical properties of the Schur method [13] and avoiding of ill-conditioned eigenvalue problems for the closed-loop state matrix $A+B F$ [5]. The reduction of the magnitudes of partial feedback gains in the Schur method can be achieved in several ways. The use of an appropriate pole matching strategy during assigning of poles clearly could contribute to reduce the norm of the computed partial feedback. For example, at each step it is possible to choose the eigenvalues to be assigned in $\Gamma^{\prime}$ those which are the nearest to $\Lambda(\alpha)$, the eigenvalues of the last block to be modified. In particular, the use of this strategy ensures a zero partial feedback matrix in the case when the two eigenvalues sets coincide. In this way, the Schur method is able to produce a zero feedback matrix if the eigenvalues to be assigned are precisely the eigenvalues of $A$. Notice that none of the presently known methods is able to guarantee this feature for $m>1$. Because $\|F\| \leq \sum\left\|F_{i}\right\|$, a second way to reduce the norm of $F$ is by explicitly minimizing the norms of the partial feedback matrices. The combination of both techniques can be very efficient in determining small norm feedback matrices. Notice however that the use of minimum norm partial feedbacks does not guarantee a minimum norm of the final feedback.

In this section we consider the problem to compute a minimum norm feedback $F$ to solve the $\mathrm{EAP} \Lambda(A+B F)=$ $\Gamma$ for $n=1$ or $n=2$. We consider only the Frobenius norm, but unfortunately even in this case explicit formulas can not be derived for the entries of the minimum norm feedback for $n=2$. The case $n=1$ with $\Gamma=\{\gamma\}$ has been already discussed in the previous section. The minimum norm feedback is given by (4). We can see that

$$
\|F\|_{F}=\frac{|\gamma-A|}{\|B\|_{F}}=\frac{|\gamma-A|}{\beta},
$$

where $\beta$ is the nonzero singular value of $B$.
In the case $n=2$ we will assume without loosing from generality, that $m=2$ and the matrices $A$ and $B$, after the application of an appropriate orthogonal state similarity and input coordinate transformations, have the forms

$$
A=\left[\begin{array}{ll}
\alpha_{11} & \alpha_{12} \\
\alpha_{21} & \alpha_{22}
\end{array}\right], \quad B=\left[\begin{array}{cc}
\beta_{1} & 0 \\
0 & \beta_{2}
\end{array}\right],
$$

where $\beta_{1} \geq \beta_{2}$ are the singular values of $B$. The case $m=$ 1 is recovered by setting $\beta_{2}=0$ and the case $m>2$ can be reduced to the case above by compressing the columns of $B$ with the help of the singular value decomposition. Because of controllability assumption $\beta_{1}>0$ and either $\beta_{2}>0$ or if $\beta_{2}=0$ then $\alpha_{21} \neq 0$. If $\Gamma=\left\{\gamma_{1}, \gamma_{2}\right\}$, then with the explicit expressions for the components of the first row row of the feedback matrix $F=\widetilde{F}$ in (6), we have to solve the unconstrained two-dimensional minimization problem

$$
\min \left\{f\left(\varphi_{21}, \varphi_{22}\right)\right\}
$$

for

$$
f:=\|F\|_{F}^{2}=\varphi_{11}^{2}+\varphi_{12}^{2}+\varphi_{21}^{2}+\varphi_{22}^{2}
$$

Unfortunately this minimization problem has no analytic solution. To solve it, gradient search methods are appropriate. Because both the gradient and the Hessian of $f$ can be explicitly computed, the Newton method is appropriate to solve this problem provided a good initial approximation $\left(\varphi_{21}^{*}, \varphi_{22}^{*}\right)$ can be used. Such an initial approximation can be computed for instance, by minimizing separately the sums of squares of the diagonal and off-diagonal terms.

Consider first the one-dimensional minimization problem for the diagonal entries of $F$

$$
\min \left\{\varphi_{11}^{2}+\varphi_{22}^{2}\right\} \text {. }
$$

The minimal gains $\varphi_{22}^{*}$ and the corresponding $\varphi_{11}^{*}$ (see (6)) are given by

$$
\varphi_{11}^{*}=\frac{\beta_{1}\left(\sigma-\alpha_{11}-\alpha_{22}\right)}{\beta_{1}^{2}+\beta_{2}^{2}}, \quad \varphi_{22}^{*}=\frac{\beta_{2}\left(\sigma-\alpha_{11}-\alpha_{22}\right)}{\beta_{1}^{2}+\beta_{2}^{2}} .
$$

By using the above computed gains, we solve next a similar one-dimensional minimization problem for the offdiagonal entries

$$
\min \left\{\varphi_{12}^{2}+\varphi_{21}^{2}\right\}
$$

to compute the minimum gain $\varphi_{21}^{*}$ and the corresponding $\varphi_{12}^{*}$ (see (6)). If we denote $\widehat{\alpha}_{11}=\alpha_{11}+\beta_{1} \varphi_{11}^{*}$ and $\widehat{\alpha}_{22}=$ $\alpha_{22}+\beta_{2} \varphi_{22}^{*}$ and if we replace in the expression of $\varphi_{12}$ in (6) $\sigma$ by $\widehat{\alpha}_{11}+\widehat{\alpha}_{22}$ and $\alpha_{22}$ by $\widehat{\alpha}_{22}$, then $\varphi_{21}^{*}$ results as the least magnitude real root of the fourth order polynomial equation

$$
\begin{aligned}
0= & \beta_{1}^{2} \beta_{2}^{3} z^{4}+3 \beta_{1}^{2} \beta_{2}^{2} \alpha_{21} z^{3}+3 \beta_{1}^{2} \beta_{2} \alpha_{21}^{2} z^{2}+ \\
& \left(\beta_{1}^{2} \alpha_{21}^{3}-\beta_{2}^{2} \alpha_{12} c+\beta_{2}^{2} \alpha_{12}^{2} \alpha_{21}\right) z+\beta_{2} c\left(\alpha_{12} \alpha_{21}-c\right),
\end{aligned}
$$

where $c=\pi-\widehat{\alpha}_{11} \widehat{\alpha}_{22}+\alpha_{12} \alpha_{21}$. With the computed initialization, the Newton method applied to minimize (11) usually converges to the minimum norm feedback in 1-2 iterations. It can be easily seen that $F=0$ if $\Gamma=\Lambda(A)$. Moreover, $\varphi_{21}=0$ and $\varphi_{22}=0$ if $\beta_{2}=0$. 


\section{Minimum Norm Feedback Stabilization}

The parametric Schur method can be easily converted into an algorithm to solve the following stabilization problem: determine $F$ such that $\Lambda(A+B F) \subset \mathbb{C}_{g}$, where $\mathbb{C}_{g}$ is an appropriate stability domain of the complex plain. We will discuss two choices for $\mathbb{C}_{g}$, namely $\mathbb{C}_{g}=\{\lambda \in \mathbb{C} \mid$ $\operatorname{Re},(\lambda) \leq s\}$ in case of a continuous-time system and $\left.\mathbb{C}_{g}=\{\lambda \in \mathbb{C}|| \lambda) \mid \leq s\right\}$ in case of a discrete-time system. The problem which we actually discuss is how to choose the poles to be assigned to achieve the desired stabilization with minimum norm partial feedbacks. Although there is no guarantee for a global minimum norm feedback, it is expected that the local pole assignment strategy to deliver global feedbacks which are usually very near to the minimum norm feedback. The case $n=1$ has the obvious solution described in the previous section where $\Gamma=\{s\}$. In the case $n=2$ we assume $A$ and $B$ have the forms in (10). The single-input case is covered by $\beta_{2}=0$.

In the continuous-time case we assume that $A$ has only complex eigenvalues. It is to be expected that the minimum norm feedback will correspond to eigenvalues lying on the boundary of $\mathbb{C}_{g}$, that is we can choose $\Gamma=$ $\{s+i y, s-i y\}$. Thus, given $s$ we try to determine the optimal value of $y, y_{\text {opt }}$, which minimizes the norm of $F$ assigning $\Gamma$. Equivalently, we can determine the optimal product $\pi_{o p t}=s^{2}+y_{o p t}^{2}$ corresponding to a given sum $\sigma=2 s$. With the explicit expressions for the components of the first row of the feedback matrix $F=\widetilde{F}$ in (6), we have to solve the unconstrained three-dimensional minimization problem

$$
\min \left\{f\left(\pi, \varphi_{21}, \varphi_{22}\right)\right\}
$$

for $f$ as in (11).

From the optimality conditions we get after straightforward but tedious formula manipulations the following expression for the minimum norm feedback

$$
F=\left[\begin{array}{cc}
\frac{\beta_{1}\left(2 s-\alpha_{11}-\alpha_{22}\right)}{\beta_{1}^{2}+\beta_{1}^{2}} & 0 \\
0 & \frac{\beta_{2}\left(2 s-\alpha_{11}-\alpha_{22}\right)}{\beta_{1}^{2}+\beta_{1}^{2}}
\end{array}\right] .
$$

The closed-loop eigenvalues are $s \pm i y_{\text {opt }}$, where

$$
\begin{aligned}
y_{\text {opt }}= & {\left[-s^{2}-\alpha_{12} \alpha_{21}-\left(\alpha_{22}-\left(\alpha_{11}+\alpha_{22}\right) c\right)^{2}\right.} \\
& \left.+2 s\left(\alpha_{22}-\left(\alpha_{11}+\alpha_{22}\right) c\right)(1-2 c)\right]^{1 / 2},
\end{aligned}
$$

where $c=\beta_{2}^{2} /\left(\beta_{1}^{2}+\beta_{2}^{2}\right)$.

In the discrete-time case the stabilizability condition can be expressed as $\pi \leq s^{2}$. With the assumption that the optimal closed-loop eigenvalues lie on the circle with radius $s$, we determine the elements of the feedback matrix as functions of the sum of eigenvalues $\sigma$ and of two further parameters, say the components $\varphi_{21}$ and $\varphi_{22}$ of the feedback $F=\widetilde{F}$ in (5). We have to solve again an unconstrained three-dimensional minimization problem

$$
\min \left\{f\left(\sigma, \varphi_{21}, \varphi_{22}\right)\right\}
$$

for $f$ as in (11).

No analytical solution exists for the above minimization problem, thus iterative gradient techniques, as for example the Newton method, can be used to find the minimizing solution. A good initialization of the Newton method is by taking $\varphi_{21}=0, \varphi_{22}=0$ and the corresponding optimal value of $\sigma$

$$
\sigma_{\text {opt }}=\frac{\alpha_{22}^{3}+\alpha_{12} \alpha_{22} \alpha_{21}+s^{2} \alpha_{22}+\alpha_{11} \alpha_{21}^{2}+\alpha_{22} \alpha_{21}^{2}}{\alpha_{22}^{2}+\alpha_{22}^{2}} .
$$

\section{Computation of Minimum Frobenius-Norm Feedback}

In [4] an approach has been proposed to determine a minimum Frobenius-norm feedback $F$ to assign a set of poles $\Gamma$. This approach relies on the following parametrization of the EAP: given the parameter matrix $G \in \mathbb{R}^{m, n}$ and the matrix $\widetilde{A} \in \mathbb{R}^{n, n}$ such that $\Lambda(\widetilde{A})=\Gamma$, determine $F$ as $F=G X^{-1}$, where $X$ satisfies the Sylvester equation

$$
A X-X \widetilde{A}+B G=0 .
$$

Usual restrictions on choosing $\widetilde{A}$ and $G$ are: 1) the pair $(\widetilde{A}, G)$ is observable; 2$) \Lambda(A) \cap \Lambda(\widetilde{A})=\emptyset$. If additionally the pair $(A, B)$ is controllable, then $X$ satisfying (12) is generically invertible and $F$ solves the EAP because $X^{-1}(A+B F) X=\widetilde{A}$. Although redundant, this parametrization has the advantage to allow the use of standard minimization procedures to compute the minimum norm feedback. By defining the performance index

$$
J=\frac{1}{2}\|F\|_{F}=\frac{1}{2} \operatorname{tr}\left(F^{T} F\right),
$$

its gradient with respect to $G$ can be computed by employing the formulas derived in [4] as

$$
\nabla_{G} J=H^{T}-B^{T} U^{T},
$$

where $H=X^{-1} F^{T}$ and $U$ satisfies the Sylvester equation

$$
\widetilde{A} U-U A-H F=0 .
$$

Assuming $\widetilde{A}$ in RSF, the solution of this equation in the evaluation of the gradient involves about $5 n^{3} \div 6 n^{3}$ operations if the Hessenberg-Schur method of [1] is employed.

Because of condition $\Lambda(A) \cap \Lambda(\widetilde{A})=\emptyset$, this approach does not allow partial eigenvalue assignment. In what follows we propose a new approach which eliminates this inconvenience and additionally drastically reduces the cost of evaluating gradients. Our approach relies on the following observation: the performance index $J$ is invariant to an 
orthogonal transformation, that is $J=\frac{1}{2} \operatorname{tr}\left(\widehat{F}^{T} \widehat{F}\right)$, where $\widehat{F}=F Q$ with $Q$ an orthogonal matrix. If $F$ is the minimum norm feedback for the pair $(A, B)$ then $\widehat{F}$ is the minimum norm feedback for the pair $(\widehat{A}, \widehat{B})=\left(Q^{T} A Q, Q^{T} B\right)$. Thus we can first reduce $A$ to RSF which involves only once about $10 n^{3}$ operations and then evaluate the gradient for the reduced pair $(\widehat{A}, \widehat{B})$ with $\widehat{A}$ in RSF. The solution of the corresponding reduced Sylvester equation (13) involves this time only $n^{3}$ operations. Further, to allow for partial pole assignment, the following trick can be used. By using an orthogonal similarity transformation, reduce the matrix $A$ to the ordered RSF (1), where $A_{11} \in \mathbb{R}^{q, q}$, $\Lambda\left(A_{11}\right) \subset \mathbb{C} \backslash \mathbb{C}_{b}$ and $\Lambda\left(A_{22}\right) \subset \mathbb{C}_{b}$. Let us partition

$$
Q^{T} B=\left[\begin{array}{c}
B_{1} \\
B_{2}
\end{array}\right]
$$

accordingly. Now we can compute by using gradient search techniques the minimum norm solution $F_{2}$ of a reduced order EAP such that $\Lambda\left(A_{22}+B_{2} F_{2}\right)=\Gamma_{n-q}$. The final minimum norm feedback results as $F=\left[\begin{array}{ll}0 & F_{2}\end{array}\right] Q^{T}$.

Remark. Notice that the achieved minimum norm of $F$ depends also on the choice of the matrix $\widetilde{A}$. Thus there is no guarantee that for arbitrary $\widetilde{A}$, the resulting minimum norm $F$ has the least possible norm.

\section{Conclusion}

Parameterized pole assignment offers supplementary possibilities to achieve additional features in designing state feedback control laws. A general purpose parametric Schur method using a non-redundant set of parameters has been proposed. Several possibilities have been investigated to compute minimum norm feedback matrices. An improved approach to compute minimum Frobenius-norm feedback has been also developed relying on a redundant parametrization of the EAP. All proposed computational approaches are easily implementable using available standard numerical software.

\section{References}

[1] G. H. Golub, S. Nash, and C. Van Loan. A Hessenberg-Schur method for the problem $A X+$ $X B=C$. IEEE Trans. Autom. Control, 24:909-913, 1979 .

[2] N. J. Higham. Optimization by direct search in matrix computations. SIAM J. Matrix Anal. Appl., 14:317-333, 1993.

[3] J. Kautsky, N. K. Nichols, and P. Van Dooren. Robust pole assignment in linear state feedback. Int. J. Control, 41:1129-1155, 1985.

[4] L. H. Keel, J. A. Fleming, and S. P. Bhattacharyya. Minimum norm pole assignment via Sylvester's equa- tion. In Linear Algebra and Its Role in Systems Theory, volume 47 of AMS Contemporary Mathematics, pages 265-272. 1985.

[5] V. L. Mehrmann and H. Xu. An analisis of the pole placement problem. I. The single input case. Preprint SFB393/95-35, Technical University Chemnitz, Nov. 1995.

[6] G. S. Miminis and C. C. Paige. A direct algorithm for pole assignment of time-invariant multi-input linear systems using state feedback. Automatica, 24:343$356,1988$.

[7] R. V. Patel and P. Misra. Numerical algorithm for eigenvalue assignment by state feedback. Proc. IEEE, 72:1755-1764, 1984.

[8] P. H. Petkov, M. M. Konstantinov, D. W. Gu, and I. Postletwaite. Optimal eigenstructure assignment of linear systems. Technical report, 93-64, Department of Engineering, Leicester University, UK, 1993.

[9] P. Hr. Petkov, N. D. Christov, and M. M. Konstantinov. A computational algorithm for pole assignment of linear multiinput systems. IEEE Trans. Autom. Control, AC-31:1755-1764, 1986.

[10] J. Sreedhar and P. Van Dooren. Pole placement via the periodic Schur decomposition. In Proc. 1993 American Control Conference, San Francisco, CA, pages 1563-1567, 1993.

[11] P. Van Dooren. The generalized eigenstructure problem in linear systems theory. IEEE Trans. Autom. Control, AC-26:111-129, 1981.

[12] A. Varga. A Schur method for pole assignment. IEEE Trans. Autom. Control, AC-26:517-519, 1981.

[13] A. Varga. The numerical stability of an algorithm for pole assignment. In G. Leininger, editor, Proc. 2-nd IFAC CADCS Symp., West Lafayette, Indiana. Pergamon Press, Oxford, 1982.

[14] A. Varga. A Schur method for computing coprime factorizations with inner denominators and applications in model reduction. In Proc. 1993 American Control Conference, San Francisco, CA, pages 21302131, 1993.

[15] A. Varga. Generalized Schur methods to compute coprime factorizations of rational matrices. In Proc. 1st Asian Control Conference, Tokyo, Japan, volume 3, pages 89-92, 1994.

[16] A. Varga. On stabilization of descriptor systems. Systems \& Control Letters, 24:133-138, 1995. 